\title{
TEMPO NECESSÁRIO PARA NOVILHAS NELORE CRIADAS A PASTO, GANHAREM 210, 240, 270 KG DESDE O NASCIMENTO
}

\author{
(Days from birth to get 210, 240, $270 \mathrm{~kg}$ for nellore heifers on grazing)
}

\author{
ROSA, A.B. ${ }^{1}$; SOUZA, J.C. ${ }^{2}$; SILVA, L.O.C. ${ }^{3}$; PEREIRA, E.B. ${ }^{1}$; MALHADO, C.H.M.4; FERRAZ FILHO, \\ P.B. ${ }^{5}$; GONDO, A. ${ }^{6}$; FREITAS, J.A. ${ }^{2}$
}

\begin{abstract}
1'Acadêmica de Zootecnia - AG/DZ - UFPR - Rua dos Funcionários, 1540 - Curitiba - PR CEP 80035050 - aislanna@ufpr.br -Orientada do segundo autor. ${ }^{2}$ Prof. e Pesquisador do Campus Palotina - UFPR, PR; ${ }^{3}$ Pesquisador da Embrapa Gado de Corte, MS; ${ }^{4}$ Prof. Assistente UFSBA - BA;

${ }^{5}$ Prof. e Pesquisador do Departamento de Ciências Naturais - DCN - UFMS - MS; ${ }^{6}$ Analista de Sistemas - Embrapa Gado de Corte, MS.
\end{abstract}

\begin{abstract}
RESUMO - O objetivo foi avaliar o número de dias para novilhas da raça Nelore (4.577) ganharem 210,240 e $270 \mathrm{~kg}$ a partir do nascimento, criadas em regime de pasto. Para as análises estatísticas, utilizou-se o método dos quadrados mínimos. O modelo continha os efeitos fixos de mês e ano de nascimento da novilha e a idade da mãe como covariável; como efeito aleatório, touro aninhado dentro de fazenda e o erro. As respostas mostraram que é possível desafiar fêmeas mais jovens, com pesos menores, quando lhes são asseguradas boas condições nutricionais, e assim permitir que seu crescimento não seja interrompido.
\end{abstract}

Palavras chaves: desempenho, produção, Zebu.

ABSTRACT - The objective of this study was to evaluate the number of days for 4,577 nellore heifers raised in regimen of grass to get 210,240 and $270 \mathrm{~kg}$ of weight since the birth. For statistical analyses, the square means method was used. The model contained the fixed effects from helfer's month and year of birth and the dam's age as co-variable; as random effect, bull sheltered inside the farm and the error. The results showed that it is possible to challenge younger females, with lower weight, when good nutritional conditions are assured, allowing uninterrupted growth.

Key words: performance, production, Zebu.

\section{Introdução}

O Brasil possui o segundo maior rebanho de bovinos e o maior rebanho comercial do planeta, com aproximadamente 185 milhões de cabeças (IBGE, 2003), o que representa 16\% do rebanho do globo. Os diversos sistemas de produção que são utilizados no país fazem com que os índices de produtividade do rabanho nacional sejam baixos. Esta produtividade é afetada por diversos parâmetros (tais como: intervalo de partos, idade ao primeiro parto, idade de abate, taxa de fertilidade, etc.) que na sua maioria devem ser melhoradas afim de minimiza-las fazendo com que o potencial real de produção seja explorado.
TONHATI e SIMIONI (2004) avaliando a relação genética entre a idade ao primeiro parto e primeira data do parto na raça Nelore, verificaram que a idade ao primeiro parto apresenta grande interesse zootécnico, pois retrata o inicio do processo reprodutivo e produtivo das fêmeas, ou seja, quanto mais rápido a fêmea ganha determinado peso, mais cedo ela estará pronta para entrar na fase reprodutiva e maior será o número de bezerros produzidos por ela. De acordo com GRESSELER (1998), a idade ao parto é uma indicação de precocidade, visto que aquelas que emprenham mais cedo, parem mais cedo. O acompanhamento do desenvolvimento da vida produtiva do animal permite ao criador 
saber qual vaca esta produzido e assim, elevar seus ganhos econômicos.

Segundo GIANNONI e GIANNONI (1989), as duas principais maneiras de se avaliar a velocidade de crescimento, desde que as condições ambientais sejam corrigidas para todos os indivíduos em controle, são: a contagem do número de dias necessários para o animal ganhar determinado peso e a verificação do ganho de peso num período fixo. FRIES et al. (1996) lançou a idéia de se utilizar como critério de seleção dias para o animal ganhar determinado peso. Tal proposta seria uma re-expressão do ganho médio diário (GMD) dos animais. SILVA et al. (2003), estudando dias para fêmeas da raça Guzerá ganharem $270 \mathrm{~kg}$ de peso verificou que os animais atingiam a meta com média de 599,3 $\pm 6,8$ dias de idade. Ainda no mesmo trabalho constatou-se o efeito significativo de touro dentro de fazenda (aleatório), dos efeitos fixos de ano e mês de nascimento da novilha e idade da vaca ao parto (linear e quadrático).

O objetivo do presente trabalho foi avaliar o número de dias para novilhas da raça Nelore ganharem 210, 240 e $270 \mathrm{~kg}$ a partir do nascimento, sendo as mesmas criadas em regime de pasto.

\section{Material e Método}

Os dados são provenientes do convênio $A B C Z$ / CNPGC - EMBRAPA, composto de 4.577 novilhas da raça Nelore, criadas em regime de pasto em 118 fazendas localizadas no estado de Mato Grosso do Sul. As novilhas são filhas de 437 touros, nascidas nos doze meses do ano, no período compreendido entre 1976 e 2001. Os pesos foram ajustados por interpolação entre dois pesos consecutivos de um mesmo animal, com base nas informações do banco de dados. O número de dias para ganhar determinado peso foi calculado com a seguinte fórmula:

$$
\mathrm{Dp}=\frac{\text { peso kg }}{\mathrm{GND}}
$$

Onde $\mathrm{Dp}$ é o número dias para ganhar determinado peso $(210,240$ e $270 \mathrm{~kg})$; peso no numerador, é uma variável que assumiu os valores 210,240 ou 270 , de acordo com a característica estudada; e, GND, o ganho de peso no período desejado, do nascer à idade avaliada. Os animais que apresentaram ganhos inferiores a $0,100 \mathrm{~kg}$, por terem uma idade bastante elevada quando atingiram os respectivos pesos, foram descartados, por serem considerados inaptos para seleção.

As análises estatísticas foram realizadas utilizando-se o método dos quadrados mínimos, através de procedimento GLM do programa SAS, 2002. O modelo estatístico continha os efeitos fixos de mês e ano de nascimento da novilha e a idade da vaca como co-variável (linear e quadrática); como efeito aleatório, utilizou-se touro aninhado dentro de fazenda e o erro.

Para as estimativas dos componentes de covariância, assim como o cálculo das herdabilidades e das correlações genéticas, ambientais e fenotípicas, foi utilizado o método da Máxima Verossimilhança Restrita utilizando o software MTDFREML. O modelo estatístico para estimativa dos componentes de variância e parâmetros genéticos continha os efeitos aleatórios aditivo direto e materno (ambiente permanente de vaca), bem como os efeitos fixos de grupo de contemporâneos (fazenda, mês e ano de nascimento do animal) e da covariável idade da vaca ao parto (efeitos linear e quadrático).

\section{Resultados e Discussão}

A análise de variância revelou efeito significativo de todas as fontes de variação do modelo, com exceção da idade da vaca ao parto, que foi não-significativo (TABELA 1). 
TABELA 1 - RESUMO DA ANÁLISE DE VARIÂNCIA DE DIAS PARA GANHO DE 210, 240 E 270 KG E DOS PESOS AOS 415, 470 E 530 DIAS DE IDADE EM NOVILHAS DA RAÇA NELORE, MATO GROSSO DO SUL. $(n=4577)$.

\begin{tabular}{|c|c|c|c|c|c|c|c|}
\hline \multirow[b]{2}{*}{ FV } & \multirow[b]{2}{*}{ GL } & \multicolumn{6}{|c|}{ Quadrados médios } \\
\hline & & D210 & P415 & D240 & P470 & $\mathrm{D} 270$ & P530 \\
\hline$\overline{\mathrm{NP}}$ (Faz) & 840 & 2055,11 * & * $1455,83^{* *}$ & $2658,82^{* *}$ & $6783,04^{* *}$ & $3364,68^{* *}$ & $828,66^{* \star}$ \\
\hline MN & 11 & $4495,87 *$ & 4316,30 ** & $5448,39 * *$ & $2891,05 *$ & 6783,04 ** & 1546,10 ** \\
\hline NA & 25 & 1584,78 & 1914,40 * & 2298,79 ** & 739,94 & $2891,05 * *$ & 621,19 * \\
\hline IV & 1 & $449,63 n$ & $157,43 \mathrm{~ns}$ & $533,39 \mathrm{~ns}$ & $1562,18 \mathrm{~ns}$ & $739,94 \mathrm{~ns}$ & $115,59 \mathrm{~ns}$ \\
\hline iv*iv & 1 & $1012,68 \mathrm{~ns}$ & $55,81 \mathrm{~ns}$ & $1214,37 \mathrm{~ns}$ & $3364,68 \mathrm{~ns}$ & $1562,18 \mathrm{~ns}$ & $130,23 \mathrm{~ns}$ \\
\hline Erro & 3698 & 733,55 & 797,72 & 939,45 & 402,53 & 1178,37 & 356.35 \\
\hline Total & 4576 & - & - & - & - & - & - \\
\hline
\end{tabular}

$\mathrm{NP}(\mathrm{faz})$ = número do pai aninhado dentro de fazenda; $\mathrm{MN}$ = mês de nascimento, $\mathrm{AN}$ = ano de nascimento; IV = idade da vaca linear; IV*IV = idade da vaca, quadrática; D210 = número de dias para ganhar $210 \mathrm{~kg}$; P415 = peso aos 415 dias de idade; D240 = número de dias para ganhar $240 \mathrm{~kg} ; \mathrm{P} 470$ = peso aos 470 dias de idade; D270 = número de dias para ganhar $270 \mathrm{~kg} ; \mathrm{P} 530=$ peso aos 530 dias de idade; ${ }^{*} \mathrm{P}<0,0001 ;{ }^{*} \mathrm{P}<0,05 ; \mathrm{ns}$ = não significativo.

Os $\mathrm{R}^{2}$ dos modelos utilizados nas análises de variância, para cada uma das características avaliadas, foram respectivamente 0,$405720 ; 0,408286$, 0,410369; 0,336155,0,341437, 0,363598, para D210, D240, D270 e P415, P470, P530, mostrando valores razoáveis, visto que são dados de campo. Quanto aos coeficientes de variação, na mesma seqüência, apresentaram valores iguais à 6,459609 , 6,393600, 6,361971, 11,19840, 7,337454, 6,337685 , evidenciando a presença de variabilidade genética para os pesos estudados, assim como a possibilidade de minimizar o número de dias para atingir es respectivas idades.

A média de idade estimada para as novilhas atingirem 210, 240 e $270 \mathrm{~kg}$ foram iguais a 423, 484 e 545 dias, respectivamente. Por outro lado, fêmeas nascidas nos meses de abril a julho apresentaram desempenho superior a média, portanto, foram mais tardias. As nascidas nos meses de março e agosto desempenho semelhantes a média e as demais, nascidas no período de setembro a dezembro, janeiro e fevereiro foram as que tiveram melhor desempenho, sendo o valor mínimo obtido (GRÁFICOS 1, 2 e 3). Esses resultados são importantes, principalmente para que realiza estação de monta e precisa de novilhas mais desenvolvidas e prontas para serem acasaladas no início do período.

Quanto a dias para ganhar 210, 240 e $270 \mathrm{~kg}$ de peso vivo, somados ao peso ao nascer, cuja a média das fêmeas na população avaliada foi igual a $29,80 \mathrm{~kg}$. As mesmas encontram-se com peso total igual a 239,$80 ; 269,80$ e $299,80 \mathrm{~kg}$, mostrando que a essas idades as fêmeas já podem ser colocadas com touros. Resultados semelhantes foram encontrados por SILVA et al. (2003), para D270 em animais da raça Guzerá.

Considerando a necessidade de se obter animais precoces para peso e idade, desafiar as novilhas mais novas, pode ser uma grande vantagem para melhorar o desempenho dos sistemas de produção. Estando em condições favoráveis de ambiente, os animais portadores de genótipos superiores podem manifestar seu potencial. 
GRÁFICO 1 - NÚMERO DE DIAS PARA GANHO DE 240 KG EM FUNÇÃO DO MÊS DE NASCIMENTO, EM BOVINOS DA RAÇA NELORE. MATO GRSSO DO SUL. ( $n=4577)$.

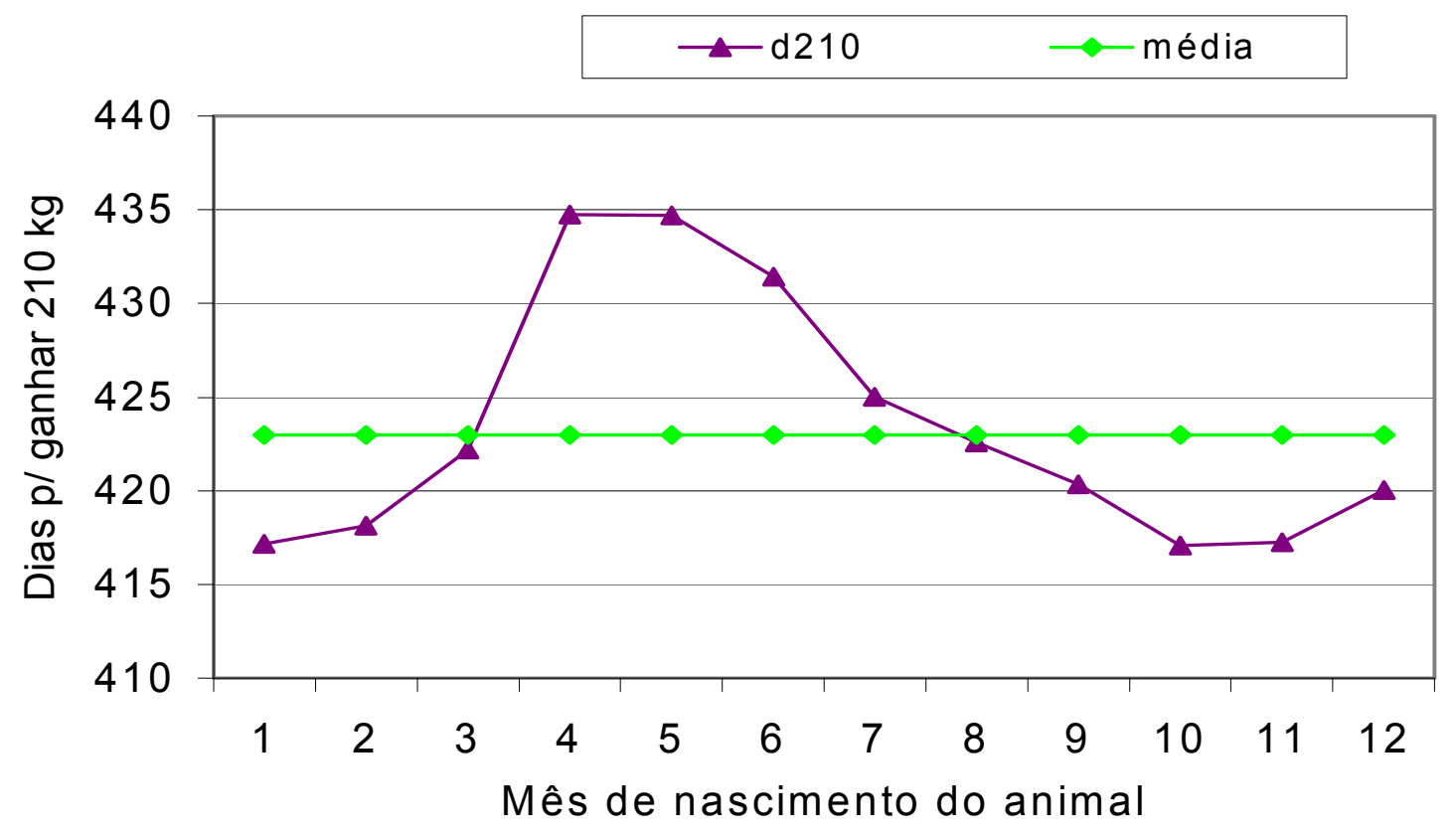

GRÁFICO 2 - NÚMERO DE DIAS PARA GANHO DE 240 KG EM FUNÇÃO DO MÊS DE NASCIMENTO, EM NOVILHAS DA RAÇA NELORE. MATO GRSSO DO SUL. $(n=4577)$.

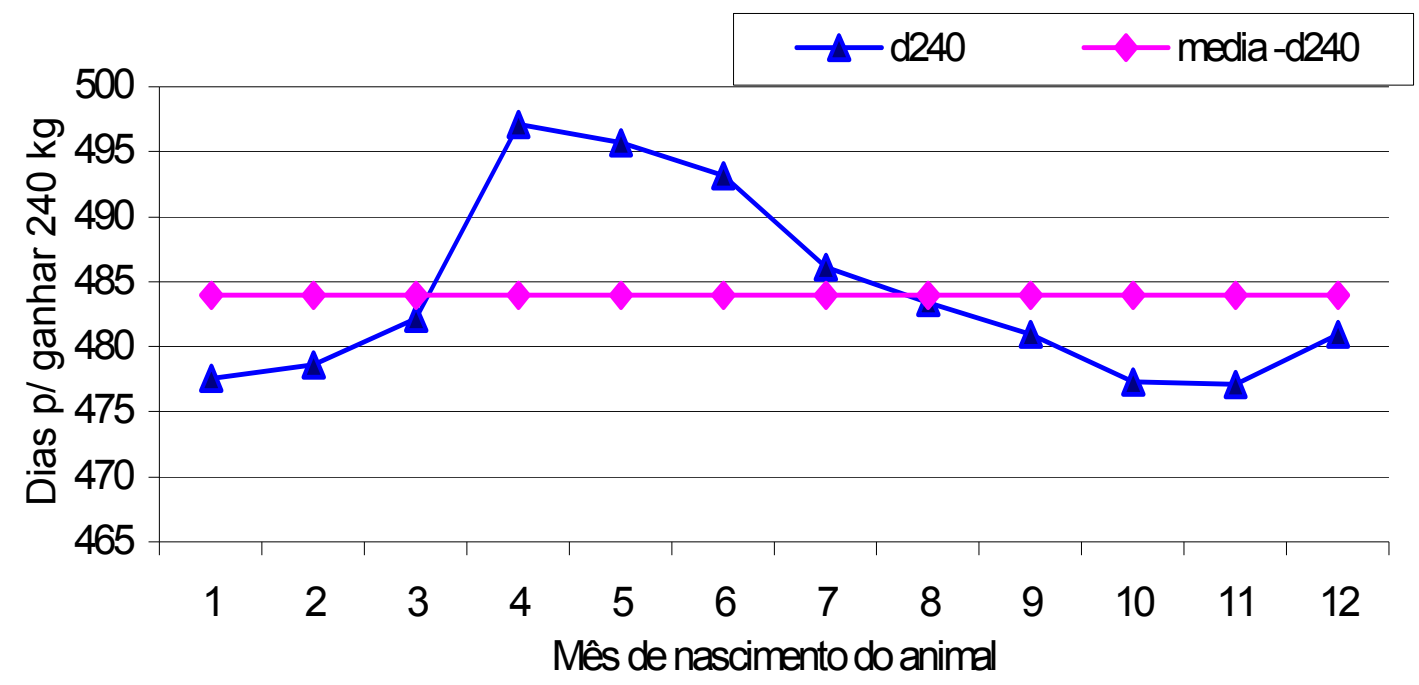


GRÁFICO 3 - NÚMERO DE DIAS PARA GANHO DE 270 KG EM FUNÇÃO DO MÊS DE NASCIMENTO, EM NOVILHAS DA RAÇA NELORE. MATO GROSSO DO SUL. $(n=4577)$.

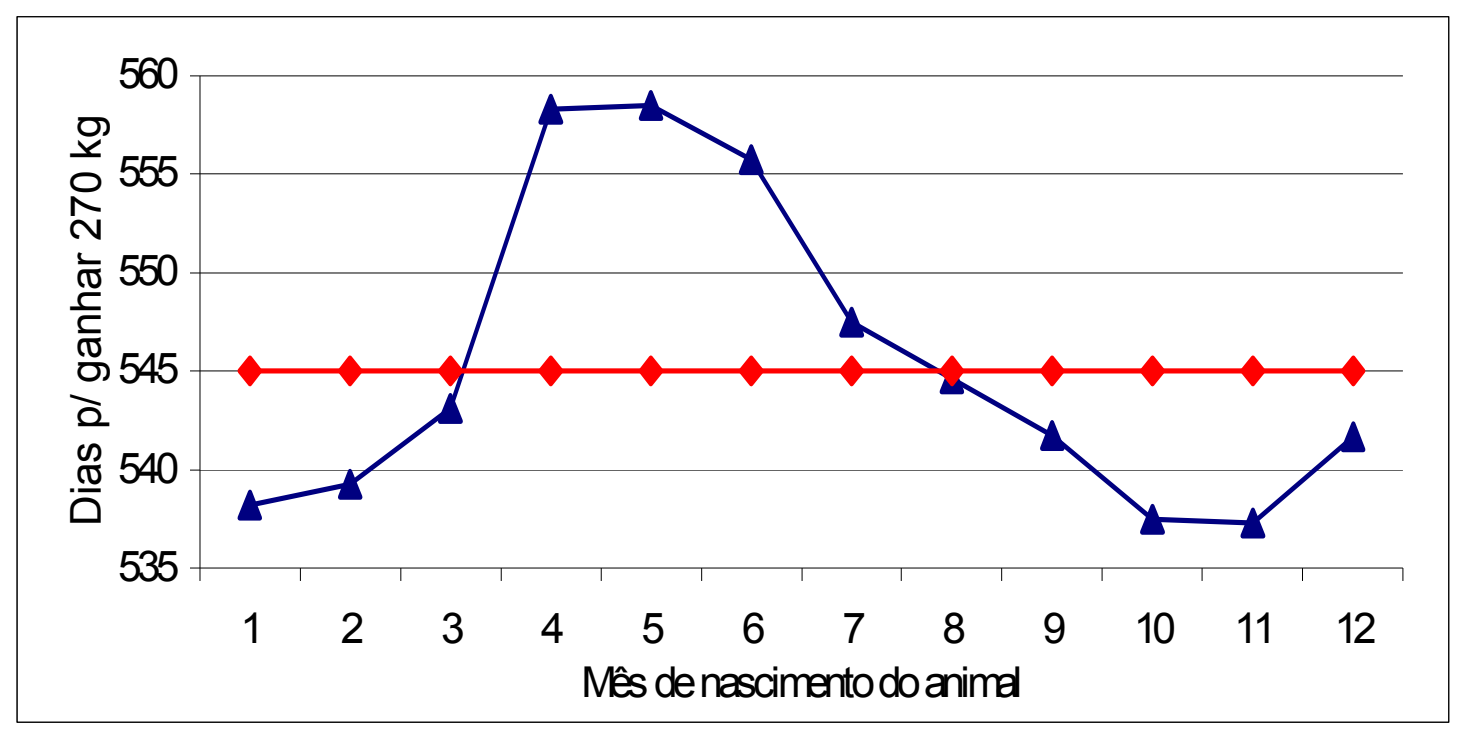

Assim, mesmo sendo inseridas em grupos de acasalamento, com peso abaixo do mínimo, as novilhas continuam em crescimento, ganhando peso, e têm condições de sair da estação de monta prenhes com o peso mínimo desejado. Isso permite que aquelas novilhas que se encontravam com peso um pouco abaixo do desejado, para que elas entrassem na estação de monta, participe da mesma. Dessa forma, as mesmas não terão que esperar mais um ano vazias, para conceber. Porém, é importante salientar a necessidade de haver eficácia (não deixar faltar) na oferta de alimentos para as mesmas, durante e após a estação de monta para que não haja interrupção de seu desenvolvimento, e conseqüentemente, promover um maior intervalo de parto entre o primeiro e segundo parto da mesma.

Às estimativas dos componentes de variância e parâmetros genéticos, encontram-se na TABELA 2.

TABELA 2 - VARIÂNCIA GENÉTICA, FENOTÍPICA E AMBIENTAL E HERDABILIDADE DA CARACTERÍSTICA DIAS PARA GANHO DE 210, 240 E 270 KG A PARTIR DO NASCIMENTO, EM NOVILHAS DA RAÇA NELORE, MATO GROSSO DO SUL. $(n=4577)$.

\begin{tabular}{lccccc}
\hline & $\sigma^{2}{ }_{A}$ & $\sigma^{2}{ }_{P}$ & $\mathrm{C}^{2}$ & $\sigma^{2}{ }_{\mathrm{e}}$ & $\mathrm{h}^{2}$ \\
\hline D210 & 64,29 & 688,22 & 39,86 & 584,08 & $0,09 \pm 0,06$ \\
D240 & 86,72 & 898,62 & 53,13 & 758,77 & $0,10 \pm 0,06$ \\
D270 & 166,36 & 1119,47 & 51,27 & 901,84 & $0,15 \pm 0,06$ \\
\hline
\end{tabular}

$\sigma_{\mathrm{A}}^{2}=$ Variância aditiva direta; $\sigma_{\mathrm{P}}^{2}=$ variância fenotípica total; $\mathrm{c}^{2}=$ efeito de ambiente permanente de vaca; $\sigma_{e}^{2}=$ variância devido ao ambiente; $h^{2}=$ Herdabilidade. 
Constatou-se variabilidade genética para as características estudadas, porém, os coeficientes de herdabilidades poderiam ter sido mais elevados. Tais valores sugerem que há influências do meio sobre as variáveis aqui avaliadas. Isso evidência a importância de praticar melhoramento com boas técnicas de manejo, visto que o sistema de produção é tão frágil quanto o elo mais frágil da cadeia.

\section{Conclusões}

As fontes de variações touro dentro de fazenda, mês e ano foram significativas, mostrando ser relevantes à correção para essas fontes de variação no momento da seleção das novilhas.

Os resultados mostraram que é possível desafiar fêmeas mais jovens com pesos menores, permitindo assim identificar as fêmeas mais precoces e então selecioná-las para isso.

Por fim, com base nos resultados da influencia do mês de nascimento sobre o desempenho das fêmeas verificou-se que se criar estações de acasalamento específicas pode vir a promover ganhos ao criador.

\section{REFERÊNCIAS}

FRIES, L.A.; BRITO, F.V.; ALBUQUERQUE, L.G. Possíveis conseqüências de seleção para incrementar pesos as idades-padrão vs reduzir idade para produzir unidades de mercado. In: REUNIÃO ANUAL DA SOCIEDADE BRASILEIRA DE ZOOTECNIA, 33., 1996, Fortaleza. Anais... Fortaleza: Sociedade Brasileira de Zootecnia, 1996. p.310-312.

GIANNONI, M.A.; GIANNONI, M.L. Genética e melhoramento de rebanhos nos trópicos. 2. ed. São Paulo: Nobel, 1989. 463p.

GRESSELER, S.C. Estudo de fatores de ambiente e parâmetros genéticos de algumas características reprodutivas em animais da raça nelore. Belo Horizonte, 1998. Dissertação (Mestrado) - Universidade Federal de Minas Gerais.

INSTITUTO BRASILEIRO DE GEOGRAFIA E ESTATÍSTICA (IBGE). São Paulo, 2003.

SAS - Statistical Analysis System. User Guide: Stat, Cary, (NC: SAS INSTITUTE INC.) 2002.

SILVA, E.R.; SOUZA, J.C.; SILVA, L.O.C.; PEROTTO, D; MALHADO, C.H.M.; FERRAZ FILHO, P.B.; FREITAS. Number of days needed for Guzerath heifers gain $270 \mathrm{~kg}$ from birth. In: WORLD CONFERENCE ON ANIMAL PRODUCTION, 9., 2003, Porto Alegre. Anais... Porto Alegre: ALPA, 2003. CD-ROM.

TONHATI, H.; SIMIONI, V.M. Reação genética entre idade ao primeiro parto e primeira data do parto na raça nelore. In: REUNIÃO ANUAL DA SOCIEDADE BRASILEIRA DE ZOOTECNIA, 41, 2004, Campo Grande, MS. Anais... Campo Grande: SBZ, 2004.

Recebido para publicação: 30/01/2005 Aprovado: 